\title{
A NEW SOFT FOCUS SCREEN FOR PORTRAIT PHOTOGRAPHY.
}

BY

\section{W. FREDERICK.}

IN portrait work it is not always desirable that a lens should give sharp definition because marks and blemishes are brought out too clearly. Lines and similar defects must be smoothed out to resemble more nearly what the casual eye sees under ordinary white light. This is usually done by retouching the negative, or by employing a lens with soft definition, or both. Soft definition may be obtained in many ways, but its character makes a great difference in the appearance of a picture. It may be produced by merely throwing the lens out of focus, but the effect is not pleasing because the definition is obliterated too completely. We want something that will leave the main features of the picture clearly sketched and at the same time smooth out or obliterate unimportant or disfiguring details.

The usual method of producing soft definition is to modify the design of the lens so that it will fall off in spherical aberration. Thus the image formed by light passing through an outer zone of the lens will not be at the same distance as that formed by light passing through the central portion of the lens, and intermediate images will be formed at intermediate points, so within a certain range a part of the light will form a sharp image and the remainder a series of superposed fuzzy images. The effect aimed at is definition with a mellow outline.

It may be readily understood that the character of the effect will depend upon the distribution of light in the sharp and the fuzzy images, which distribution may be influenced by lens type and different adjustments as to spherical aberration. But the nature of spherical aberration places certain limitations upon the problem. We can not, for example, adjust the aberration of a lens so that three-fourths of the light will form a sharp image and the remaining one-fourth a diffuse image; thus we are cut off from many effects that may be desirable. It usually happens that there is too much light in the diffuse images, so that double lines appear in certain portions of the picture where there is a great 
change from white to black, as a white collar against a black coat, or a well-lighted cheek against a dark background:

The natural distribution of light due to spherical aberration may be modified by interposing an opaque diaphragm with a small central opening to allow the sharp image to be formed and radial openings to control the amount of light in the diffuse image. But such a device has the disadvantage of slowing down the lens to an inconvenient degree. However, it was a study of the action of such a diaphragm that suggested the new diffusing screen which is the subject of this paper.

The necessity for a means of diffusion independent of the lens arose in connection with a new enlarging camera which was being developed. This camera was fitted with a device which kept the lens automatically in focus as the enlargement was varied. It was to be used in enlarging portraits, and it was necessary that both sharp definition and soft definition could be obtained at will. It would have been an easy matter to design a mount for the lens which by unscrewing one component would alter the relative positions of the lens elements and introduce spherical aberration. But this would cause a change in the focal length of the lens, and any change in this would prevent its proper functioning. Thus it was necessary to find something independent of the lens.

What at first appeared to be a hardship led eventually to emancipation. Many things were thought of and tried. Diffraction was invoked and rejected. Semitransparent material such as cloth and ground glass were tried but found to slow down the lens too much. Glass slides, coated with transparent varnish giving an undulatory surface, failed to prove satisfactory because the diffusion was not controlled, some of the light being scattered so far that the entire picture was flattened.

We were finally led to try glass discs with fine grooves polished in them. If the grooves were accurately cut the diffusion would be controlled so that no ray of light could deviate more than a limited angle, a few minutes, from its undisturbed path, and thus the picture would not be flattened by stray light. Of course the smoothing out of fine detail will give a certain appearance of flattening which can not be avoided, but this is not noticeable in portrait photography. The discs were placed directly in front of the lens, and being entirely transparent caused no slowing down of the combination. 
A great many experiments were tried with different devices for cutting the grooves, different depths and widths of grooves, and different patterns cut upon the disc. The possibilities were endless, and each change had an individuality of its own in its effect upon a photograph.

First we tried concentric circular grooves cut upon the outer portion of the glass disc, leaving the central portion clear for about half the aperture of the lens. The light passing through the central portion of the screen would give a sharp definition, and that passing through the outer portion of the screen diffuse definition. Then by merely stopping the lens the amount of light in the diffuse image could be reduced, and the relative proportion of light in the sharp and diffuse images altered at will. But this screen proved unsatisfactory because it was found to give double lines along boundaries of strong contrast, there being too much light in the rays of maximum deviation. Also the circular grooves were hard to cut.

To get around these difficulties we changed to cylindrical grooves ground in intersecting linear patterns. It was expected the intersecting grooves would diminish the area of maximum slopes, and thus reduce the amount of light of maximum diffusion. But this, too, was a failure, as it was found the successive systems of grooves simply superposed themselves upon the glass surface whether flat or undulating. At the intersection of two grooves the glass was abraded to twice the depth for one groove, and no advantage was obtained.

The grooves could be readily examined by means. of interference fringes. When the ground surface of one disc was placed in contact with the unground surface of another, interference bands would appear, giving a very beautiful contour map of the whole system of cuts. When seen under a mercury lamp these contour lines became very sharp indeed, making it easily possible to estimate the depth of the cuts to one or to two millionths of an inch.

Finally, after many experiments, a screen was settled upon in which there were three systems of linear grooves ground at angles of one hundred and twenty degrees from each other, and the grooves of each system were spaced at equal intervals apart but were progressively of increasing depth from the centre outward. The finished pattern showed hexagonal areas of clear glass 
constituting about two-thirds of the area of the entire screen. Thus two-thirds of the light was allowed to form a sharp image, and the remaining one-third a succession of soft images varying in diffuseness according to the depth of the successive grooves of the screen. The screen gives soft effects without a trace of double lines, and at the same time it gives definition that does not fail under a magnifier. Its effect may be slightly modified by stopping down the lens.

The results obtained with this screen were very good indeed and distinctly better than anything that could be done with a soft focus lens. In fact, they were so promising that we hastened to apply for a patent upon the idea.*

The screens are capable of many unusual effects. One screen was ground with a single system of parallel grooves, which when used with the diffusion vertical in a portrait had a tendency to make the face appear longer, or if the diffusion were horizontal the face would appear broader. In landscapes it gave similar effects. If the diffusion were vertical the corresponding detail, such as grass or tree trunks, would remain sharp in the picture, while horizontal detail as limbs and twigs of trees would be fuzzed out. Thus it is possible that with proper care effects could be obtained similar to those seen in the paintings of Corot.

A rectangular system of grooves gives a very satisfactory screen for landscapes, and also for portraits. If placed with the diffusion at about forty-five degrees the detail of a landscape will be streaked out in a way suggestive of etching. It is possible that a rectangular system with one set of grooves deeper than the other would be found useful.

One screen of the hexagonal type was ground with one system of grooves strong and the other two weak, but we have not had time to give it a serious test. It might be used to alter the length of a face, or might be turned so as to bring out better detail in hair, or to alter the appearance of cloth in a dress or coat. A satin dress should preserve its peculiar sheen much better if the greater diffusion were directed parallel to the grain of the cloth.

A fundamental peculiarity of all the screens consisting of

* [EdrTor's Note: Unfortunately, no known printing process can reproduce the striking and beautiful effects shown by the pictures submitted by $\mathrm{Mr}$. Frederick in illustration of the work done by means of this new diffusing screen. These include a landscape and a dozen or more large portraits.] 
systems of straight lines is that the diffusion occurs at right angles to the various systems, and does not occur equally in all directions. If a small hole be pricked in a negative its image in the enlargement will be seen to consist of a point with short radial streamers extending out from it in a star-like pattern. This is probably beneficial in helping to preserve the contrast between the blacks and whites in fine detail, as one set of streamers is likely to coincide closely with the direction of the detail, thus adding to the already preponderant light in the sharp image, while subtracting nothing from the softening effect upon the picture.

While most of our efforts have been directed toward developing a diffusing screen for enlarging cameras, it is possible that they may be useful also in direct portrait photography. A few large screens for this purpose were ground at haphazard, and tried in a tentative way. The results seemed to indicate that the problem of direct taking was somewhat different from that of enlarging, and would require a different type of screen. In direct taking the screen causes the white areas to encroach upon the black, producing an effect similar to halation, while in the case of enlarging, the black areas encroach upon the white and do not produce the effect of halation. Thus in direct photography a white collar will irradiate upon a black coat and spoil the appearance of the picture, while in an enlargement the black coat will encroach upon the white collar with no unpleasant effect. However, by limiting the diffusion and modifying its distribution it is quite likely that a satisfactory screen may be developed for direct photography. At least the action of the screen in this case is similar to that of the soft focus lenses now in use, and the greater adaptability of the screen should be in its favor.

Hawk Eye Works, Eastman Kodak Company,

Rochester, N. Y., Feb., 1920. 